\title{
Phase-Contrast Magnetic Resonance Angiography Measurements of Global Cerebral Blood Flow in the Neonate
}

\author{
MANON J.N.L. BENDERS, JEROEN HENDRIKSE, LINDA S. DE VRIES, FRANK VAN BEL, AND FLORIS GROENENDAAL \\ Departments of Neonatology [M.J.N.L.B., L.S.V., F.B., F.G.] and Radiology [J.H.], Wilhelmina Children's Hospital, University Medical \\ Center, 3508 AB Utrecht, The Netherlands
}

\begin{abstract}
Cerebral blood flow $(\mathrm{CBF})$ alterations are important in pathogenesis of neonatal ischemic/hemorrhagic brain damage. In clinical practice, estimation of neonatal CBF is mostly based on Doppler-measured blood flow velocities in major intracranial arteries. Using phase-contrast magnetic resonance angiography (PC-MRA), global CBF can be estimated, but there is limited neonatal experience. The objective of this study was to gain experience with PC-MRA for the determination of global CBF in neonates. In infants eligible for MRI, PC-MRA global CBF was determined by measuring volume blood flow in both internal carotid arteries (ICAs) and basilar artery (BA). Thirty newborns (GA, 25.7-42.1 wk; weight, 1050-5858 g; postconceptional age, 225-369 d) were investigated. Total PC-MRA CBF ranged from 27 to $186 \mathrm{~mL} / \mathrm{min}$. Significant correlations between PC-MRA CBF and postconceptional age and weight were detected. When calculating PC-MRA measured CBF per kilogram body weight, brain perfusion was about stable over the range of postconceptional ages and ranged between 11 and 48 $\mathrm{mL} / \mathrm{min} / \mathrm{kg}$ (median, $25 \mathrm{~mL} / \mathrm{min} / \mathrm{kg}$ ). In conclusion, neonatal PCMRA CBF seems to be a useful technique to estimate noninvasive CBF. (Pediatr Res 69: 544-547, 2011)
\end{abstract}

$\mathrm{P}$ evious studies of (changes in) neonatal cerebral blood flow (CBF) have been performed using different methods, such as the venous occlusion plethysmography (1), positron emission tomography (2), the nitrous oxide technique (3), and the ${ }^{133}$ Xenon clearance method (4). In addition, Xenon computed tomography (5) and more recently MRI with contrast (6) have been used to measure neonatal CBF. Furthermore, the use of near-infrared spectroscopy (NIRS) with indocyanine green has been repeatedly reported, but it uses an i.v. injection with the dye indocyanine green (7). Ionizing radiation is involved in these techniques or they are invasive or need special requirements, which limit their use. Arterial spinlabeled perfusion MRI (8) and sophisticated NIRS techniques such as optical diffuse correlation spectroscopy are developed at this moment to evaluate neonatal CBF (9); however, improvements are necessary before these devices can reliably be used. It may be clear that the above-mentioned methods are not (yet) available for clinical use in the vulnerable and unstable (preterm) neonate.

Noninvasive approaches for estimating changes in global $\mathrm{CBF}$ in the often unstable neonate are performed using NIRS

Received March 22, 2010; accepted December 14, 2010.

Correspondence: Manon Benders, M.D., Ph.D., Department of Neonatology, Wilhelmina Children's Hospital, KE 04.123.1, PO Box 85090, 3508 AB Utrecht, The Netherlands; e-mail: m.benders@umcutrecht.nl and Doppler-derived blood flow velocity measurements in the major intracranial arteries (10-14). NIRS as such has not yet become a routine application in the NICUs, whereas measurements of blood flow velocity in intracranial arteries cannot measure volume flow due to the small diameter of the feeding intracranial arteries in newborn infants $(5,12)$.

MRI is increasingly used in neonates both in preterm as well as in full term infants, mainly for depicting the various brain regions to detect subtle differences or early ischemic and/or hemorrhagic changes due to perinatal complications $(15,16)$. Recently, using phase-contrast magnetic resonance angiography (PC-MRA), we performed measurements of total global CBF in millimeters per minute $(\mathrm{mL} / \mathrm{min})$ in a relatively large cohort of term equivalent preterm neonates. The aim of this study was to assess the anatomy of the circle of Willis in prematurely born infants at term-equivalent age and to evaluate whether these anatomic variations have an effect on blood flow through the internal carotid arteries (ICAs) and the basilar artery (BA) (17). This article together with another article that described PC-MRA in two neonates after extracorporal membrane oxygenation highlighted the potential use of PC-MRA for determination of global CBF by measuring $\mathrm{CBF}(\mathrm{mL} / \mathrm{min})$ in both ICAs and BA in the newly born infant $(17,18)$. PC-MRA measurements at the skull base are noninvasive and fast to perform with a scan time of less than a minute providing us the volume flow in $\mathrm{mL} / \mathrm{min}$ (17).

PC-MRA has not been applied in larger series of newborns, and no data are available for a wider range of postconceptional age and/or weight. It is known from Doppler ultrasound studies that the flow in the ICAs and BA is related to GA (19). With the more widespread use of MRI in neonates admitted to NICUs, this relatively simple and fast PC-MRA measurement may add to a better understanding of the pathophysiology of the neonatal cerebral perfusion as has been the case in adults $(20-22)$.

The purpose of this study was therefore to assess the feasibility of PC-MRA measurements in neonates with different postconceptional ages admitted to the NICU to obtain an indication about the magnitude of global CBF in neonates and to assess the influence of postconceptional age and weight on brain perfusion.

Abbreviations: BA, basilar artery; CBF, cerebral blood flow; ICA, internal carotid artery; PC-MRA, phase-contrast magnetic resonance angiography 


\section{METHODS}

Patients. Thirty preterm and full-term infants, all appropriate for GA and consecutively admitted to our NICU, who needed an MRI investigation for various clinical reasons, were included in the study. About half of the infants were on mechanical ventilation during the MRI.

The study was approved by the local Medical Ethics Committee. Written informed parental consent was always obtained for those infants included in the study.

$\boldsymbol{P C}-\boldsymbol{M R A}$. All MRIs were performed on a 1.5-T Philips Intera Imager (Philips Medical Systems, Best, The Netherlands) using a sense head coil. After the conventional T1- and T2-weighted images, the 2D phase-contrast (2D-PC) MRA was performed. On basis of a localizer MRA slab in the sagittal plane, a 2D-PC section was positioned at the base of the skull to measure the volume flow in the ICAs and the BA. Figure $1 A$ illustrates the positioning of the 2D-phase contrast section through both ICAs and BA (repetition time, $16 \mathrm{~ms}$; echo time, $9 \mathrm{~ms}$; flip angle $7.5^{\circ}$; slice thickness, 5 $\mathrm{mm}$; field of view, $250 \times 250 \mathrm{~mm}$; matrix size, $256 \times 256 ; 8$ averages; velocity sensitivity, $30 \mathrm{~cm} / \mathrm{s})(17,20-22)$. Quantitative flow volume values in $\mathrm{mL} / \mathrm{min}$ were calculated in each vessel by integrating across manually drawn regions of interest that enclosed the vessel lumen closely with Philips software on the MR system. Previously, we (J.H. and F.G.) have reported that this method is accurate to calculate the flow values in full-term infants, with an intraobserver variability of $5.6 \%$ and an interobserver variability of $5.5 \%$ $(17,18)$. To calculate the total $\mathrm{CBF}$, the quantitative flow volume values of both ICAs and the BA (Fig. $1 B$ ) were summed.

The infants were sedated during the imaging procedure to prevent motion artifacts. A neonatologist was always present during the examination. During the MRI/PC-MRA procedure, the neonates were placed in a vacuum fixation pillow (Med Vac Infant Immobilizer Bag; CFI Medical Solutions, Fenton, MI). Monitoring was performed using pulse oximetry (Nonin, Minneapolis, $\mathrm{MN}$ ), and respiration rate was observed using the standard Philips equipmen (Philips Medical Systems, Best, The Netherlands). For hearing protection, Minimuffs (Natus Medical Incorporated, San Carlos, CA) were used.

Statistical analysis. Clinical data are summarized as median and ranges. Correlations between PC-MRA measured global CBF on the one hand and postconceptional age and actual weight on the other hand were determined using simple linear regression analysis. The correlation coefficients are shown for descriptive purposes only. To investigate whether changes in PC-MRA measured global CBF has been affected by two important actual clinical conditions (mechanical ventilation and abnormal head circumference [lower than 10th percentile according to Dutch charts]) and by gender, multiple linear regression analysis (MLR) has been performed with PC-MRA measured global $\mathrm{CBF}$ in $\mathrm{mL} / \mathrm{min} / \mathrm{kg}$ as the dependent variable $(Y)$. An equation for the dependent variable PC-MRA was solved using sequentially the independent variables expressed as a set of dummy variables for yes/no mechanical ventilation, male/female gender, and normal/abnormal head circumference using the effects coding technique (23). A more detailed explanation of the analysis has been given elsewhere (24). The regression equation was

$$
Y=b_{\mathrm{O} 1}+b_{\mathrm{MV}}+b_{\mathrm{G}}+b_{\mathrm{HC}},
$$

where $Y$ is the dependent variable (PC-MRA measured CBF per kilogram body weight); $b_{\mathrm{O} 1}$ (the intercepts of the equations) is its overall mean; and $\mathrm{MV}, \mathrm{G}$, and $\mathrm{HC}$ represent the dummy variables for the additional effects
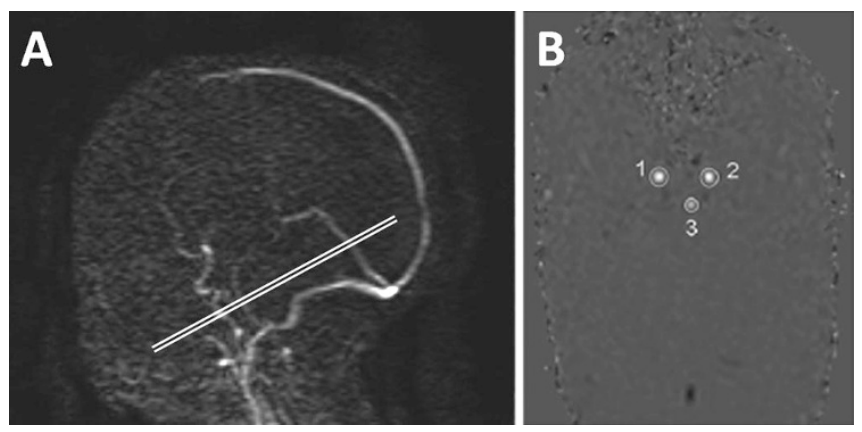

Figure 1. Illustration of the position at the base of the skull of the $2 \mathrm{D}$ phase (sagittal) contrast section through the ICAs and the BA for measurement of the volume flow of these arteries $(A)$. The numbers in the axillar plane indicate the localization of the respective vessels: both ICAs (1 and 2) and the BA (3); whereas in the far field, the sinus rectus is shown without a number (black spot) $(B)$. of mechanical ventilation, gender, and head circumference, respectively. The " $b$ " associated with each (dummy) variable represents its coefficients. To determine the statistical significance of any variable, an $F$ test was performed by dividing the mean square of that variable by the mean square residual.

Statistical significance was assumed for $p<0.05$. Statistical analysis was performed with SPSS for windows (SPSS version 12.0).

\section{RESULTS}

Clinical characteristics. Table 1 shows the clinical data of the infants included in the study. Eighteen infants were male and 12 female. Fifteen infants were mechanically ventilated during the investigations. Ten infants were born after a pregnancy of less than $32 \mathrm{wk}$. The indication for the MRI/ PC-MRA investigation in this subgroup was participation in a research protocol. Three infants had a periventricular and/or intraventricular hemorrhage; five infants received hydrocortisone treatment because of bronchopulmonary dysplasia; one infant had a small subdural effusion; and one infant suffered from perinatal asphyxia. Four infants were prematurely born between 32 and 37 wk: three infants had an intraventricular hemorrhage and one infant suffered from perinatal asphyxia. The remaining 16 infants were full term and the MRI/PCMRA indications varied from seizures to perinatal asphyxia, suspicion of perinatal stroke, postextracorporeal membrane oxygenation, suspicion of congenital infection, and subdural hematoma. The postnatal age during MRI/PC-MRA investigation ranged from $7 \mathrm{~d}$ up to $133 \mathrm{~d}$ with a median of $23 \mathrm{~d}$. Investigations were performed between 32 and 53 postconceptional weeks (median, $41 \mathrm{wk}$ ). A minority was performed earlier, in particular in the preterm population and a small minority up to 3 mo postterm, which can also be extracted from the range in postconceptional age. Median and range of head circumference percentile during the MRI/PC-MRA procedure were $\mathrm{P}_{50}$ and $\mathrm{P}_{2}$-to- $\mathrm{P}_{98}$, respectively. Only two infants had a head circumference below the 10th percentile.

PC-MRA determined global CBF (mL/min). PC-MRA measured $\mathrm{CBF}$ ranged from 27 to $186 \mathrm{~mL} / \mathrm{min}$ with a median of $91 \mathrm{~mL} / \mathrm{min}$. There was, as expected, a delineated positive correlation with postconceptional age and actual weight as can be seen in Figure $2 A$ and $B$ (open circles) showing the correlation plots $(r=0.73, p<0.0001$ and $r=0.67, p<$ 0.0001 , respectively). When calculating PC-MRA measured CBF per kilogram body weight, brain perfusion is quite stable over the whole range of postconceptional ages and ranged between 11 and $48 \mathrm{~mL} / \mathrm{min}$ with a median of $25 \mathrm{~mL} / \mathrm{min}$ as also indicated in Figure $2 A$ and $B$ (black crosses). MLR analysis revealed that mechanical ventilation, gender, and abnormal head circumference did not independently influence PC-MRA measured global CBF per kilogram.

Table 1. Important clinical characteristics

\begin{tabular}{lcr}
\hline \multicolumn{1}{c}{ Clinical data $(n=30)$} & Range & Median \\
\hline Gestational age (wk) & $25.7-42.1$ & 34.7 \\
Birth weight $(\mathrm{g})$ & $600-4460$ & 2338 \\
Postnatal age during PC-MRA (d) & $2-133$ & 123 \\
Actual weight during PC-MRA (g) & $1050-5858$ & 3500 \\
Head circumference during PC-MRA $(\mathrm{cm})$ & $29.0-40.7$ & 34 \\
\hline
\end{tabular}



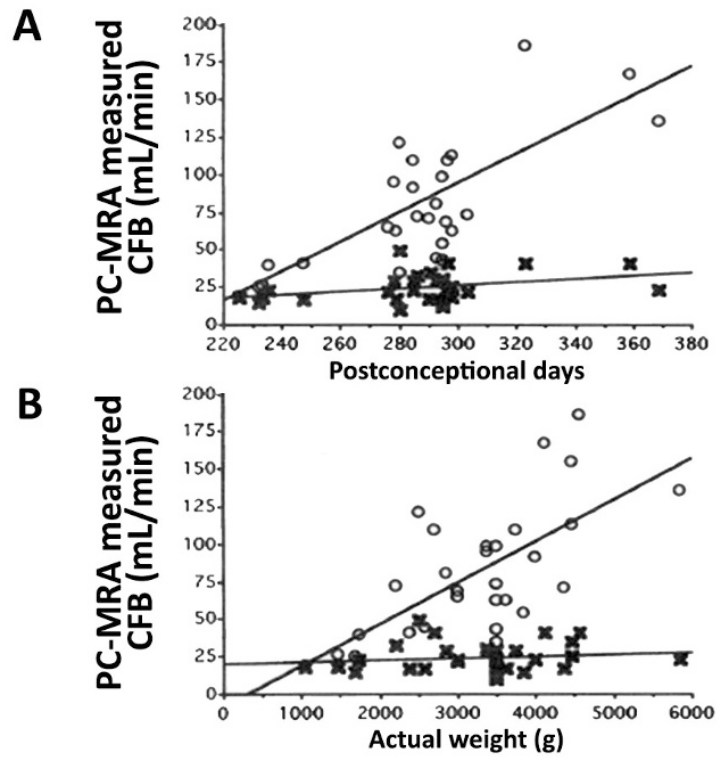

Figure 2. Correlation histogram between PC-MRA determined global blood flow as a function of postconceptional age in weeks $(A \bigcirc ; r=0.73, p<$ $0.0001)$ and as a function of actual weight in grams $(B \bigcirc ; r=0.67, p<$ 0.0001). Furthermore, PC-MRA per actual weight shown as a function of postconceptional age in weeks $(A \times ; r=0.36$, NS) and as a function of actual weight in grams $(B \times ; r=0.13$, NS $)$.

\section{DISCUSSION}

This study showed a rather strong correlation between PC-MRA measured global CBF and postconceptional age and actual body weight, which is in accordance with previous data observed in an ultrasound study reporting an increase of global CBF of $6 \mathrm{~mL} / \mathrm{min}$ per postmenstrual week (19). However, the PC-MRA measured CBF per kilogram actual body weight seemed to be remarkable stable over a wide range of postconceptional ages. Our data do not confirm an earlier study in neonates, which reported that mechanical ventilation, gender, and growth retardation negatively affected ${ }^{133}$ Xenonmeasured CBF (mL/100 gm/min) (25). Because quantitative $\mathrm{CBF}$ data in newborns are rare, it is important that evidence is added to existing knowledge with this PC-MRA technique. It seems that the data fit expectations very well. Normal brain weight is $12-15 \%$ of body weight. This means that mean CBF in this study can be estimated to $\sim 20 \mathrm{~mL} / 100 \mathrm{~g} / \mathrm{min}$. This is a bit higher than existing estimates using extracranial Doppler, Xenon, and arterial spin labeling MRI (ASL) $(8,12,19)$.

Our study revealed limitations when using these PC-MRA technique to measure perfusion in neonates. First, ethical considerations prevented examination of healthy subjects. Measurements were therefore restricted to a heterogeneous group of patients who had well-established pathology. This constraint prevented a comparative study involving both healthy subjects and patients, which may have facilitated identification of perfusion abnormalities. Second, the current cohort consisted of patients with different GAs and scanned at different postmenstrual ages, impairing interpretation of timerelated changes in perfusion. Hence, we cannot report on brain maturation effects. The values generated in this study can actually not serve as reliable reference values for preterm and full-term infants during different postconceptional ages, because only a few of the infants can be classified as infants without perinatal complications. However, it seems that the above-mentioned relations of global CBF with postconceptional age and weight are also apparent in infants with or without perinatal or neonatal complications. Moreover, almost all infants had a head circumference within the normal range at the moment of the MRI/PC-MRA procedure, which suggests that brain growth was not severely interrupted. Our study showed that mechanical ventilation or gender did not have an important effect on the PC-MRA measured CBF values. These results do not confirm results of a former study investigating CBF with the ${ }^{133}$ Xenon clearance method, although those infants were more preterm infants and the range of postnatal ages was less (25).

Despite the above-mentioned limitations in our study, the combination of MRI and PC-MRA has the potency to provide the clinician with a detailed evaluation of both brain anatomy as well as cerebral hemodynamics in various pathological conditions, which may be of great value in certain clinical conditions. Especially, the little amount of time necessary to perform a PC-MRA sequence gives less distortion due to movement of the neonate, which may give rise to a decrease in image quality in anatomical images. In future, MR cerebral perfusion measurements on brain tissue level using arterial spin labeling MRI may be possible; however, this sequence takes a longer amount of time and may decrease the image quality (26-28).

In conclusion, PC-MRA of the brain feeding arteries at the base of the skull is a fast, simple, and relatively noninvasive method to measure the global CBF in (preterm) newborns additional to conventional anatomical scans. Despite the limitations of study, we believe that PC-MRA holds great promise for future studies of normal and abnormal brain function in a variety of illnesses. It is our hope that the technique of perfusion MR will both elucidate the complexities of the developing brain and help to develop more effective treatments for brain injury in the newborn infant. More data are needed to collect a robust number of reference values of global CBF in healthy term and preterm neonates.

\section{REFERENCES}

1. Leahy FA, Sankaran K, Cates D, MacCallum M, Rigatto H 1979 Quantitative noninvasive method to measure cerebral blood flow in newborn infants. Pediatrics 64:277-282

2. Volpe JJ, Herscovitch P, Perlman JM 1983 Positron emission tomography in the newborn: extensive impairment of regional cerebral blood flow with intraventricular hemorrhage and hemorrhagic intracerebral involvement. Pediatrics 72:589-601

3. Frewen TC, Kissoon N, Kronick J, Fox M, Lee R, Bradwin N 1991 Cerebral blood flow, cross-brain oxygen extraction, and fontanelle pressure after hypoxic-ischemic injury in newborn infants. J Pediatr 118:265-271

4. Greisen G, Pryds O 1988 Intravenous ${ }^{133}$ Xenon clearance in preterm neonates with respiratory distress. Internal validation of $\mathrm{CBFO}$ as a measureof global cerebral blood flow. Scand J Clin Lab Invest 48:673-678

5. Ashwal S, Scheider S, Thompson J 1989 Xenon computed tomography measuring cerebral blood flow in the determination of brain death in children. Ann Neurol 25:539-546

6. Tanner SF, Cornette L, Ramenghi LA, Miall LS, Ridgway JP, Smith MA, Levene MI 2003 Cerebral perfusion in infants and neonates: preliminary results obtained using dynamic susceptibility contrast enhanced magnetic resonance imaging. Arch Dis Child Fetal Neonatal Ed 88:F525-F530

7. Pryds O, Edwards AD 1996 Cerebral blood flow in the newborn infant. Arch Dis Child Fetal Neonatal Ed 74:F63-F69 
8. Miranda MJ, Olofsson K, Sidaros K 2006 Noninvasive measurements of regional cerebral perfusion in preterm and term neonates by magnetic resonance arterial spin labeling. Pediatr Res 60:359-363

9. Smith MA 1990 The measurement and visualisation of vessel blood flow by magnetic resonance imaging. Clin Phys Physiol Meas 11:101-123

10. Patel J, Marks K, Roberts I, Azzopardi D, Edwards AD 1998 Measurement of cerebral blood flow in newborn infants using near infrared spectroscopy with indocyanine green. Pediatr Res 43:34-39

11. Goff DA, Buckley EM, Durduran T, Wang J, Licht DJ 2010 Noninvasive cerebral perfusion imaging in high-risk neonates. Semin Perinatol 34:46-56

12. Greisen G, Johansen K, Ellison PH, Fredriksen PS, Mali J, Friis-Hansen B 1984 Cerebral blood flow in the newborn infant: comparison of Doppler ultrasound and ${ }^{133}$ Xenon clearance. J Pediatr 104:411-418

13. Hansen NB, Stonestreet BS, Rosenkrantz TS, Oh W 1983 Validity of Doppler measurements of anterior cerebral artery blood flow velocity. Correlation with brain blood flow in piglets. Pediatrics 72:526-531

14. Wolf M, Greisen G 2009 Advances in near-infrared spectroscopy to study the brain of the preterm and term neonate. Clin Perinatol 36:807-834

15. Dubois J, Benders M, Borradori-Tolsa C, Cachia A, Lazeyras F, Ha-Vinh-Leuchter R, Sizonenko SV, Warfield SK, Mangin JF, Huppi PS 2008 Primary cortical folding in the human newborn: an early marker of later functional development. Brain 131:2028-2041

16. Cowan F, Rutherford M, Groenendaal F, Eken P, Mercuri E, Bydder GM, Meiners LC, Dubowitz LM, de Vries LS 2003 Origin and timing of brain lesions in term infants with neonatal encephalopathy. Lancet 361:736-742

17. van Kooij BJ, Hendrikse J, Benders MJ, de Vries LS, Groenendaal F 2010 Anatomy of the circle of Willis and blood flow in the brain-feeding vasculature in prematurely born infants. Neonatology 97:235-241

18. Hendrikse J, de Vries LS, Groenendaal F 2006 Magnetic resonance angiography of cerebral arteries after neonatal venoarterial and venovenous extracorporeal membrane oxygenation. Stroke 37:e15-e17
19. Kehrer M, Goelz R, Krägeloh-Mann I, Schöning M 2002 Measurement of volume of cerebral blood flow in healthy preterm and term neonates with ultrasound. Lancet 360:1749-1750

20. Hendrikse J, Klijn CJ, van Huffelen AC, Kappelle LJ, van der Grond J 2008 Diagnosing cerebral collateral flow patterns: accuracy of non-invasive testing. Cerebrovasc Dis 25:430-437

21. de Boorder MJ, Hendrikse J, van der Grond J 2004 Phase-contrast magnetic resonance imaging measurements of cerebral autoregulation with a breath-hold challenge: a feasibility study. Stroke 35:1350-1354

22. van Osch MJ, Hendrikse J, Golay X, Bakker CJ, van der Grond J 2006 Non-invasive visualization of collateral blood flow patterns of the circle of Willis by dynamic MR angiography. Med Image Anal 10:59-70

23. Slinker BK, Glantz SA 1990 Missing data in two-way analysis of variance. Am J Physiol 258:R291-R298

24. Teitel DF, Klautz RJ, Steendijk P, van der Velde ET, van Bel F, Baan J 1991 The end-systolic pressure-volume relationship in the newborn lamb: effects of loading and inotropic interventions. Pediatr Res 29:473-482

25. Baenziger O, Jaggi JL, Mueller AC, Morales CG, Lipp HP, Lipp AE, Duc G, Bucher HU 1994 Cerebral blood flow in preterm infants affected by sex, mechanical ventilation, and intrauterine growth. Pediatr Neurol 11:319-324

26. Wintermark M, Sesay M, Barbier E, Birbely K, Dillon WP, Eastwood JD, Glenn TC, Grandin CB, Pedraza S, Soustiel JF, Nairai T, Zaharchuk G, Caille JM, Dousset V, Yonas H 2005 Comparative overview of brain perfusion imaging techniques. J Neuroradiol 32:294-314

27. Wolf RL, Detre JA 2007 Clinical neuroimaging using arterial spin-labeled perfusion magnetic resonance imaging. Neurotherapeutics 4:346-359

28. Wang J, Licht DJ 2006 Pediatric perfusion MR imaging using arterial spin labelling. Neuroimaging Clin N Am 16:149-167 